\title{
Transcatheter aortic valve replacement in low risk patients: a review of PARTNER 3 and Evolut low risk trials
}

\author{
Joao Braghiroli, Kunal Kapoor, Torin P. Thielhelm, Tanira Ferreira, Mauricio G. Cohen \\ Cardiovascular Division, Department of Medicine, University of Miami Miller School of Medicine and the Elaine and Sydney Sussman Cardiac \\ Catheterization Laboratory, University of Miami Hospitals and Clinics, Miami, Florida, USA \\ Contributions: (I) Conception and design: All authors; (II) Administrative support: All authors; (III) Provision of study materials or patients: All \\ authors; (IV) Collection and assembly of data: All authors; (V) Data analysis and interpretation: All authors; (VI) Manuscript writing: All authors; (VII) \\ Final approval of manuscript: All authors. \\ Correspondence to: Mauricio G. Cohen, MD. Professor of Medicine, Cardiovascular Division, Department of Medicine, University of Miami Miller \\ School of Medicine and the Elaine and Sydney Sussman Cardiac Catheterization Laboratory, University of Miami Hospitals and Clinics, 1400 NW \\ $12^{\text {th }}$ Avenue, Suite 864, Miami, Florida 33136, USA. Email: mgcohen@med.miami.edu.
}

\begin{abstract}
Transcatheter aortic valve replacement (TAVR) has become a mainstay in treatment for patients with severe aortic stenosis who are considered high-risk surgical candidates. The use of TAVR in low-risk patients with severe aortic stenosis is being explored as an alternative to surgical aortic valve replacement (SAVR). Recent results from the Medtronic Evolut Low Risk trial and the Placement of Aortic Transcatheter Valves (PARTNER) 3 trial shed light on the use of TAVR in low-risk surgical candidates. The Evolut Low Risk trial compared TAVR with a self-expanding supra-annular bioprosthesis to SAVR in 1468 patients with severe aortic stenosis who were low surgical risk. Patients with a mean age of 74 and a mean Society of Thoracic Surgeons (STS) risk score of $1.9 \%$ were randomized to either TAVR or SAVR groups. Using the composite end point of death or disabling stroke at 24 months, the study found an incidence of $5.3 \%$ in the TAVR arm and $6.7 \%$ in the surgical arm. The Evolut Low Risk trial thus concluded that TAVR was statistically noninferior but not superior to SAVR (difference, -1.4 percentage points; $95 \%$ Bayesian credible interval for the difference, -4.9 to 2.1 ; posterior probability of noninferiority, >0.999). The PARTNER 3 trial assigned 1,000 patients with severe aortic stenosis and low surgical risk to either TAVR with transfemoral placement of balloon expandable valve or SAVR. Patients with a mean age of 73 and a mean STS score of $1.9 \%$ were randomized to either TAVR or SAVR groups. With respect to the primary endpoint of composite death from any cause, stroke, or rehospitalization, the study found an occurrence of $8.5 \%$ in TAVR and $15.1 \%$ in SAVR, confirming both noninferiority and superiority in the TAVR group [absolute difference, -6.6 percentage points; $95 \%$ confidence interval (CI), -10.8 to $-2.5 ; \mathrm{P}<0.001$ for noninferiority; hazard ratio, 0.54; $95 \%$ CI, 0.37 to $0.79 ; \mathrm{P}=0.001$ for superiority]. Both the Evolut low risk trial and the PARTNER 3 trial provide evidence that the use of TAVR extends beyond the scope of high and intermediate risk surgical patients and is at the very least equivalent to SAVR in the treatment low-risk surgical candidates when using a transfemoral approach in patients without bicuspid aortic valves. In this article we provide an extensive review on the Evolute low risk and PARTNER 3 trials, including a discussion on clinically relevant outcomes.
\end{abstract}

Keywords: Transcatheter aortic valve replacement (TAVR); surgical aortic valve replacement (SAVR); PARTNER 3; Evolut low risk

Submitted Jun 12, 2019. Accepted for publication Sep 10, 2019.

doi: $10.21037 / \mathrm{cdt} .2019 .09 .12$

View this article at: http://dx.doi.org/10.21037/cdt.2019.09.12 


\section{Introduction}

Since its first description by Cribier and colleagues in 2002 (1), transcatheter aortic valve replacement (TAVR) has undergone an accelerated period of development, revolutionizing the treatment of patients with severe symptomatic aortic valve stenosis. Currently, more patients undergo TAVR than isolated surgical aortic valve replacement (SAVR) in the United States (2).

There has been an accumulation of compelling evidence over time for the safety and efficacy of TAVR compared to SAVR. This has been driven by the implementation of the multidisciplinary heart team, groundbreaking technological advances, refined procedural techniques, and robust clinical data from large, randomized control trials (3-13).

As a reflection of those results, updated European and American society guidelines recommend TAVR as the treatment of choice for patients with symptomatic severe aortic valve stenosis in prohibitive and high surgical risk cases $(14,15)$. Furthermore, in intermediate-risk patients, TAVR has been shown to be non-inferior compared to surgery $(5,12)$.

Yet, the largest population suffering from severe, symptomatic aortic stenosis consists of relatively younger and healthier patients who are considered low-risk surgical candidates (16). For decades, this cohort has been successfully treated with SAVR. In the process, surgeons have accumulated vast experience to render low mortality and stroke rates (17), thus imposing extremely high standards for any alternative approach.

At the same time, the mise en scène for a deeper investigation into the use of TAVR for low surgical risk patients has gradually been building. There have been remarkable outcomes in five-year post-procedural data in intermediate- and high-surgical risk TAVR patients. There are encouraging results from observational studies (18-20) and the NOTION trial, a small randomized control trial comparing a self-expanding TAVR platform with SAVR for low-risk patients. Together, these findings generated momentum to investigate the safety and efficacy of TAVR compared to SAVR specifically in low-risk populations.

This review discusses the results of the Placement of Aortic Transcatheter Valves (PARTNER) 3 trial and the Medtronic Evolut Low Risk Trials, which compared the balloon expandable SAPIEN valve (Edwards Lifesciences, Irvine, CA, USA) and the self-expanding supra-annular bioprosthesis Evolut (CoreValve valve-Medtronic, Minneapolis, MN, USA) versus SAVR in a low surgical risk population.

\section{Trial design}

The design of the PARTNER 3 and low risk Evolut trials was simple with 1:1 randomization to TAVR or SAVR. Both trials included computed tomography imaging substudies to assess for the presence of reduced leaflet motion. In the PARTNER 3 trial, 1,000 patients with severe AS and low surgical risk, with a mean Society of Thoracic Surgeons Predicted Risk of Mortality (STS-PROM) score of $1.9 \pm 0.7$, were randomly assigned to transfemoral TAVR with a balloon-expandable SAPIEN S3 valve or to SAVR (21). In the Evolut low risk trial, 1,468 patients with severe AS and low surgical risk (mean STS-PROM 1.9 \pm 0.7 ) were randomly assigned to TAVR with a self-expanding valve or SAVR. A total of 1,403 patients underwent the assigned procedure. Nearly all TAVR procedures were performed via the transfemoral access (99\%) (22). The results of the imaging substudies have not been reported yet.

Patients enrolled in these trials were approximately a decade younger compared to prior studies $(3,5)$, with an average age of 73 years in PARTNER 3 and 74 years in the Evolut low risk trial. Both trials focused on reporting similar parameters, which provide an opportunity to extrapolate their findings into clinical practice.

\section{Transcatheter heart valves tested in low risk patients}

\section{SAPIEN S3 valve (Edwards Lifesciences, Irvine, CA, USA)}

The SAPIEN S3 is the third generation of SAPIEN valves (Figure 1). It consists of a bovine pericardial tissue valve within a cobalt-chromium alloy stent frame. The ventricular side of the stent is covered by a polyethylene terephthalate outer skirt designed to minimize the risk of paravalvular leak (PVL). It is the only FDA-approved platform designed to be deployed by a balloon-expandable system. The valve is delivered through a $14 \mathrm{~F}(20-, 23-$, and $26-\mathrm{mm}$ valve) or $16 \mathrm{~F}$ (29-mm valve) expandable hydrophilic coated sheath (eSheath) over a guidewire after retrograde crossing of the stenotic aortic valve. In PARTNER 3, almost half of the implanted prostheses were $26 \mathrm{~mm}$, with a limited proportion of very small-sized prostheses ( $3 \%$ were $<23 \mathrm{~mm}$ ).

While multiple alternative large-bore vascular access sites have been described in the literature, the transfemoral 


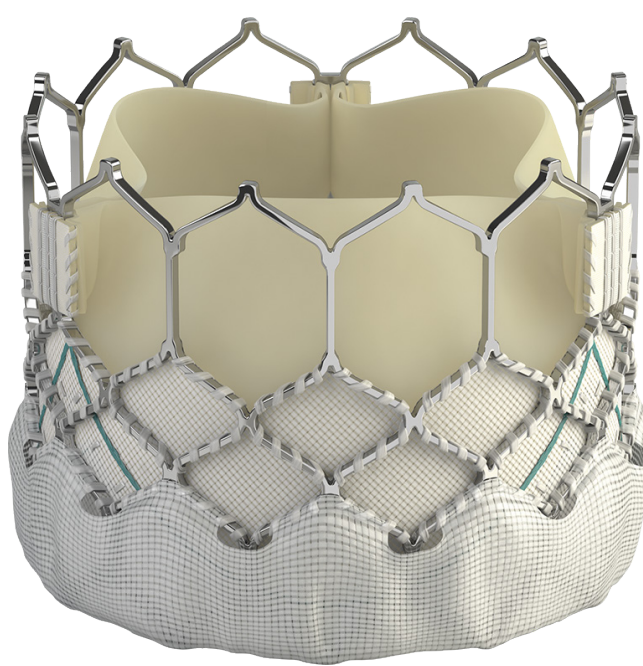

Figure 1 Edwards SAPIEN $3^{\mathrm{TM}}$ transcatheter heart valve. Permission for use of image granted by Edwards Lifesciences LLC, Irvine, CA, 2019.

approach has been shown to have lower rates of 30-day and 1 -year mortality compared with non-femoral sites (23). Of note, access was restricted to the femoral route in PARTNER 3 patients. The inability to deliver the valve from femoral access (minimum vessel diameter $<5.0 \mathrm{~mm}$ for $14 \mathrm{Fr}$ and $<5.5 \mathrm{~mm}$ for the $16 \mathrm{Fr}$ ) was an exclusion criterion. Implanted valve sizes were $20 \mathrm{~mm}(2.2 \%), 23 \mathrm{~mm}(29.2 \%)$, $26 \mathrm{~mm}(47.6 \%)$, and $29 \mathrm{~mm}(21.0 \%)$. The newer SAPIEN 3 ultra-system, which utilizes a $14 \mathrm{Fr}$ catheter, was not used in this trial.

\section{Medtronic CoreValve/Evolut R/Evolut Pro (Medtronic, Minneapolis, MN, USA)}

Three generations of Medtronic self-expanding valves were used in the study (Figure 2). The original CoreValve structure consists of a nitinol self-expanding frame housing a supra-annular porcine tissue valve. The Evolut Pro has an outer pericardial skirt that adds surface area contact between the nitinol frame and the native aortic annulus, further advancing valve sealing to prevent paravalvular regurgitation. The majority of patients received an Evolut $\mathrm{R}$ (74.1\%) or Evolut Pro (22.3\%) valve, the second and third generation CoreValve, respectively.

The Evolut platforms delivery systems have a diameter of 14 F (23-, 26-, 29 mm Evolut R) or 16 Fr (23-, 26-, 29-mm Evolut Pro and 34-mm Evolut R). A 20 F sheath was required for the CoreValve $31 \mathrm{~mm}$, the only first-

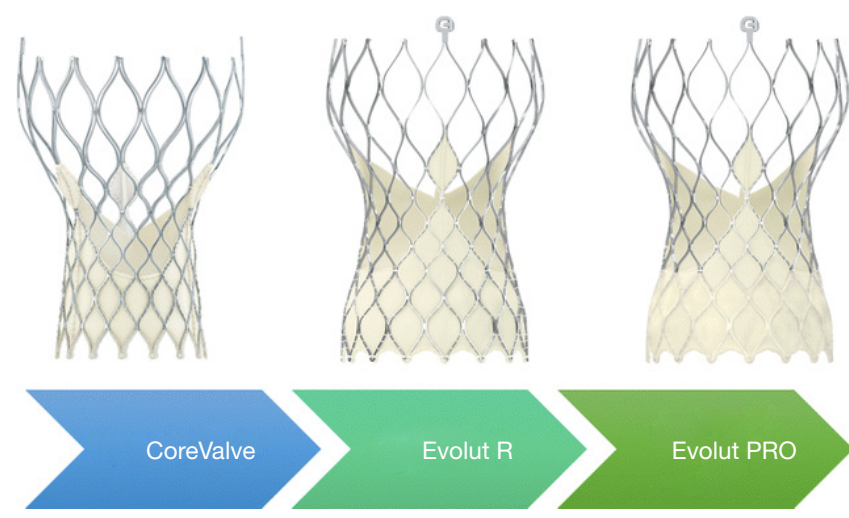

Figure 2 Medtronic(C) CoreValve TM, Evolut TM R and Evolut TM Pro Valves. Image rights obtained for use from Medtronic $₫$, 2019.

generation valve size used in $3.6 \%$ of patients in the Evolut trial. Newer generation valves include an "in-line" sheath in the delivery system that enables the operator to insert the device without the need for a larger access sheath. Implanted valve sizes were $23 \mathrm{~mm}(1.2 \%), 26 \mathrm{~mm}(19.6 \%)$, $29 \mathrm{~mm}$ (42.7\%), and 34-mm (32.9\%).

Although suboptimal candidates for transfemoral access were not excluded in the Evolut trial, the vast majority of cases did utilize this approach, with a small percentage of subclavian and direct aortic approaches (99\% transfemoral, $0.6 \%$ subclavian, $0.4 \%$ direct aortic).

\section{Procedural details}

There have been certain salient differences in procedural details between the two trials. Primarily, pre-dilatation was more frequently used with balloon expandable valves $(57.8 \%$ vs. $34.9 \%)$. In juxtaposition, the need for post-TAVR dilatation was greater with the self-expanding system $(31.3 \%$ vs. $20.9 \%)$.

Secondly, a lower percentage of patients with balloonexpanding valves received general anesthesia $(33.3 \%$ vs. $56.9 \%)$. The mean total procedure time was also significantly lower for the balloon-expandable system (58.6 min with S3 vs. 148.2 min with CoreValve), with an impressive low mean total fluoroscopic time of $13.9 \mathrm{~min}$. The higher use of general anesthesia in self-expanding valve cases may reflect differences in anesthesia practice patterns of the participating institutions, rather than specific requirements of the procedure itself, and may explain the longer procedural times. 


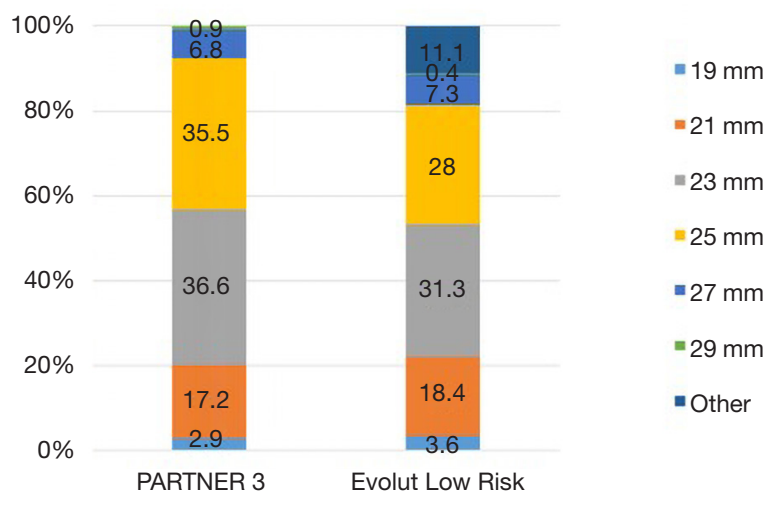

Figure 3 Surgical valve sizes.

Thirdly, a similar percentage of patients in both trials had concomitant or staged percutaneous coronary intervention ( $6.5 \%$ vs. $6.9 \%)$. Yet, revascularization was higher in the surgically treated group. A higher number of SAVR patients underwent bypass surgery, while fewer in the TAVR group underwent concomitant percutaneous coronary intervention.

\section{Surgical valves}

Specific surgical approaches were left to the discretion of the operator. For example, in PARTNER 3, a minimallyinvasive mini-sternotomy or right anterior thoracotomy incision was performed in $24.3 \%$ of patients. The distribution of surgical valve sizes was comparable, particularly in regards to the extremes of small or large annular sizes. In both trials small-diameter bioprosthetic valves $(19$ and $21 \mathrm{~mm}$ ) were utilized in approximately $20 \%$ of cases (Figure 3), with a slightly higher percentage of aortic root enlargement procedures in PARTNER 3 ( $4.6 \%$ vs. $1.6 \%$ ). The mean total surgical procedure time was higher in the Evolut Low Risk Trial (276 vs. 208 min). However, the total pump time of 97.7 and 93.4 minutes, and aortic clamp time of 74.3 and 68.7 minutes, were similar in the PARTNER 3 and Low Risk Evolut trials, respectively.

\section{Analysis of the results and implications}

The primary endpoints differed across both trials. In PARTNER 3, the primary endpoint was a composite of death from any cause, all stroke, or rehospitalization due to valve-related or procedure-related causes (including heart failure) at 12 months. In comparison, the primary endpoint in the low risk Evolut trial included all-cause mortality and disabling stroke at 24 months.

In PARTER 3 the primary endpoint was lower in the TAVR group than in the SAVR group (8.5\% vs. $15.1 \%)$. The outcome difference met the pre-specified criteria for non-inferiority and superiority ( $\mathrm{P}<0.001$ for both). Of note, the composite of death or disabling stroke at 12 months was significantly lower with TAVR than SAVR [1\% vs. 2.9\%; $\mathrm{HR}, 0.34 ; 95 \%$ confidence interval (CI): 0.12-0.97; $\mathrm{P}=0.03$ ]. Subgroup analysis demonstrated a similar benefit of TAVR across all subgroups compared to SAVR (21).

In the Evolut low risk trial, the primary composite endpoint of death or disabling stroke at 24 months occurred in $5.3 \%$ in the TAVR group and $6.7 \%$ in the SAVR group. The difference met non-inferiority, but not superiority criteria. Of note, the Evolut low risk trial did not include rehospitalization in the composite primary endpoint. Rehospitalization data was specifically reported for heart failure, and endpoints were assessed at 2 years in Evolut low risk and at 1 year in PARTNER 3.

\section{Mortality}

It is reassuring that mortality was lower in both low-risk trials compared to prior TAVR studies, as depicted in Figure 4. This may reflect the inclusion of a younger, healthier subset of patients along with utilization of improved technology and procedural techniques. In PARTNER 3, mortality at 30 days and 1 year was numerically lower with TAVR compared to SAVR $(0.4 \%$ vs. $1.1 \%$ and $1.0 \%$ vs. $2.5 \%$, respectively). In addition, all-cause mortality at 1 year was substantially reduced compared to previous studies, including higher risk patients (24.2\% in PARTNER 1 and $12.3 \%$ in PARTNER 2) (3,5). In Evolut low risk, the 30-day all-cause mortality was $0.5 \%$ with TAVR and $1.3 \%$ with SAVR. All-cause mortality at 1 year was also substantially lower in low-risk compared to moderate- and high-risk patients (22).

Yet, while overall mortality rates were numerically lower in both low-risk studies for TAVR $v s$. SAVR, after pooling these two studies in a meta-analysis including all pivotal randomized TAVR $v s$. SAVR trials, there is an aggregate relative decrease in all-cause mortality of $12 \%$ with TAVR (HR, 0.88; 95\% CI: 0.78-0.99, P=0.030) (Figure 5) (24).

\section{Stroke}

Both PARTNER 3 and Evolut low risk yielded lower stroke rates with TAVR compared to SAVR (Figure 6). In 

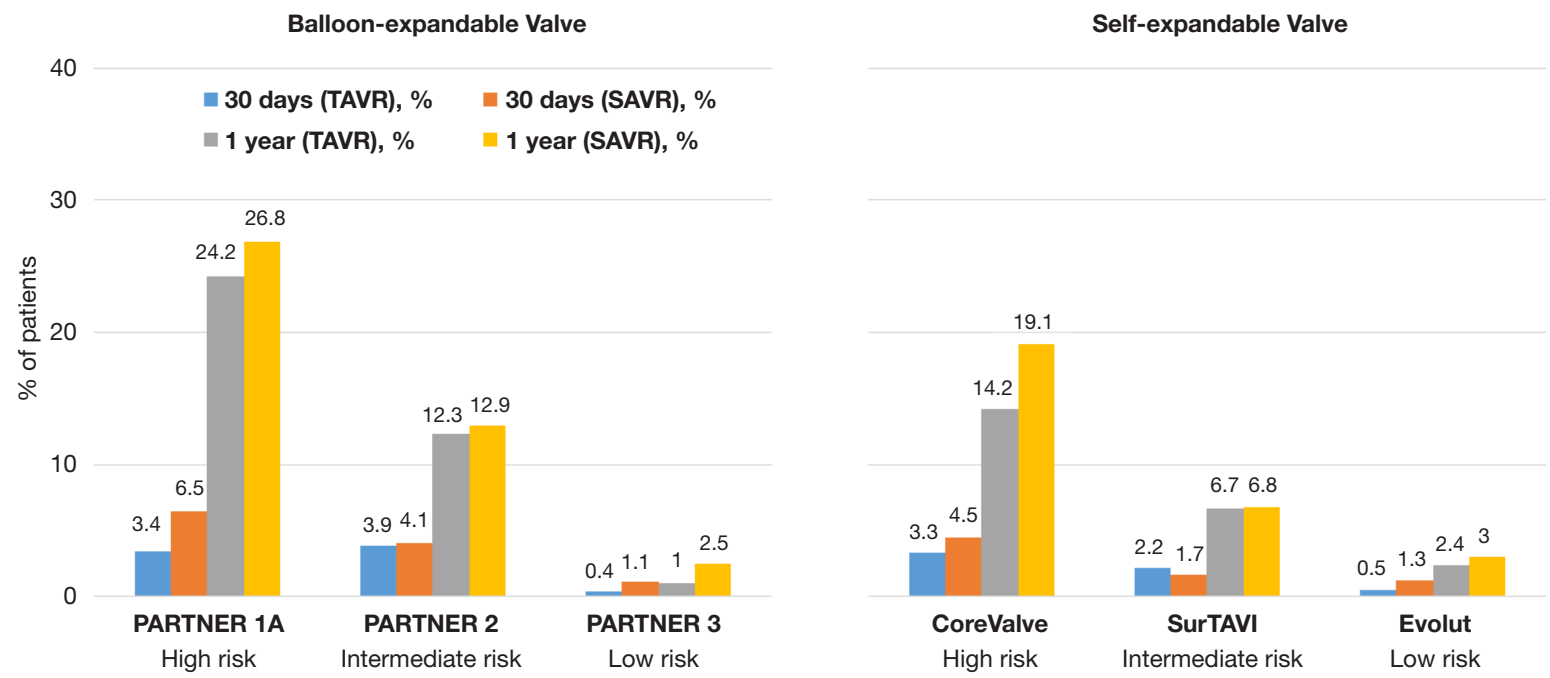

Figure 4 Surgical and TAVR mortality in clinical trials according to baseline risk. TAVR, transcatheter aortic valve replacement.

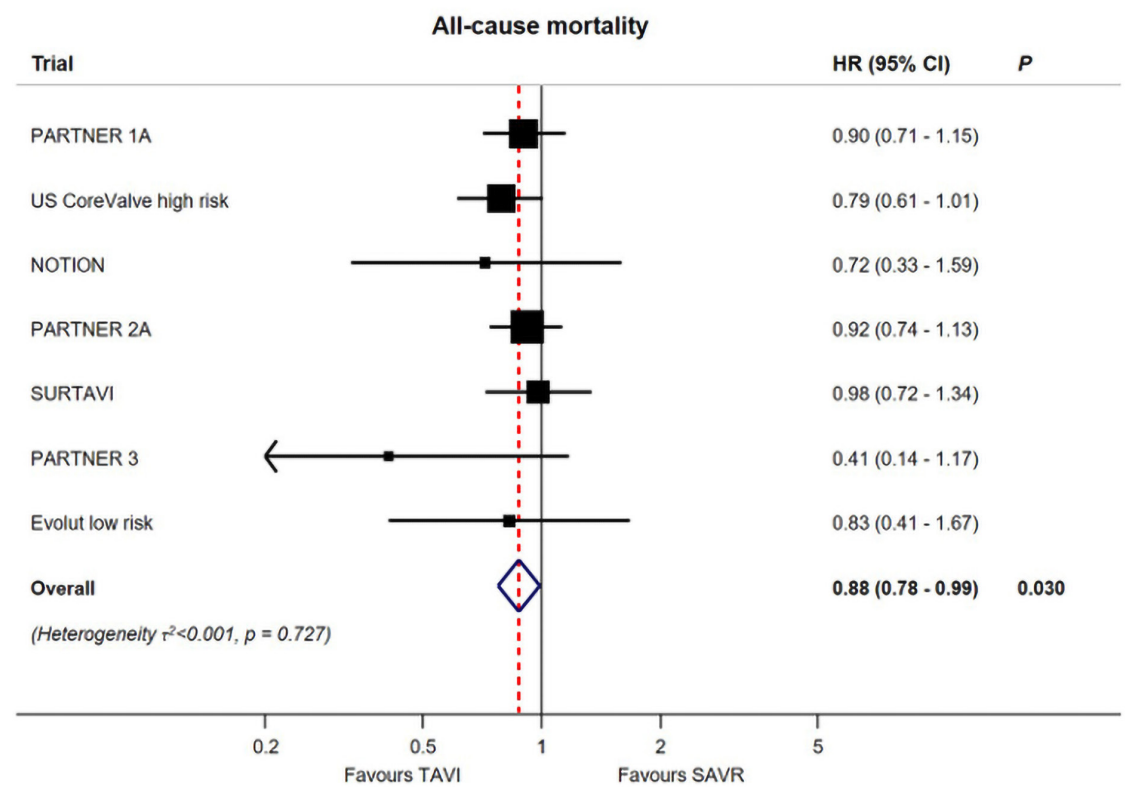

Figure 5 All-cause mortality (24).

PARTNER 3, the one-year stroke rate was $1.2 \%$ vs. $3.1 \%$, and in Evolut low risk it was $4.1 \%$ and $4.3 \%$ in the TAVR and SAVR groups, respectively $(21,22)$. In an updated metaanalysis, the data suggests a lower incidence of stroke with TAVR, but not of disabling strokes (Figure 7) (24).

Compared with previous trials in higher risk patients, the risk of stroke has decreased significantly with contemporary devices and procedural techniques that minimize manipulation of the aortic valve complex, limiting dislodgment of debris and embolization (25). With the inception and adoption of cerebral protection devices, it is expected that stroke risk will decrease even further (26). In addition, each transcatheter heart valve may possess a device-specific risk of stroke. A comparative study assessing the debris captured by the Sentinel device amongst SAPIEN 3, Evolut R, and the Lotus valves showed that balloon-expandable valves were associated with larger debris, whereas the Lotus valve was associated with the 


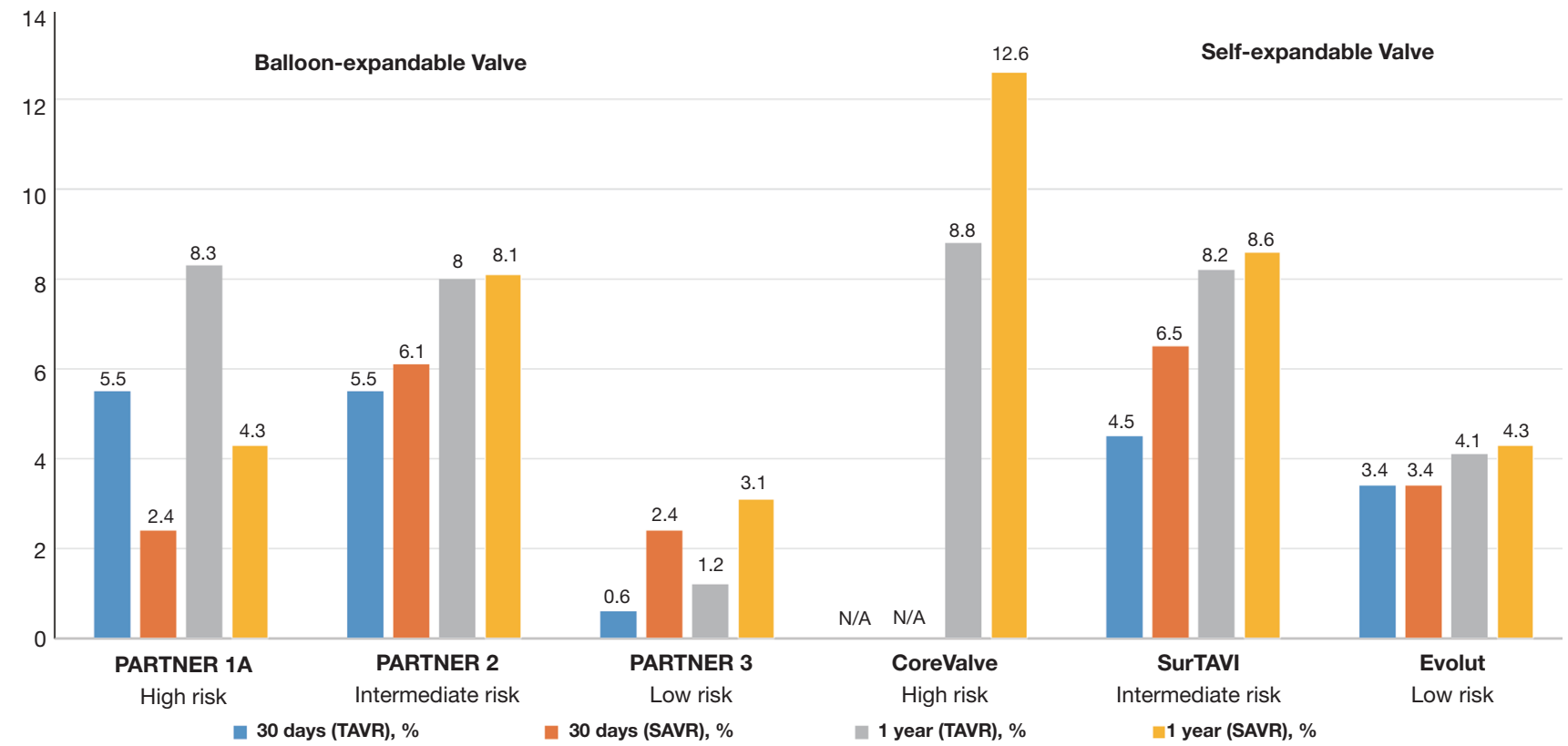

Figure 6 Stroke rate. N/A, not available.

Any- and disabling stroke

Trial
Any stroke
PARTNER 1A
US CoreValve high risk
NOTION
PARTNER 2A
SURTAVI
PARTNER 3
Evolut low risk
Overall
(Heterogeneity $\left.\tau^{2}<0.001, p=0.416\right)$
Disabling stroke
PARTNER 1A
US CoreValve high risk
PARTNER 2A
SURTAVI
PARTNER 3
Evolut low risk
Overall
(Heterogeneity $\left.{ }^{2}=0.094, p=0.097\right)$

Figure 7 Stroke, meta-analysis (24). 


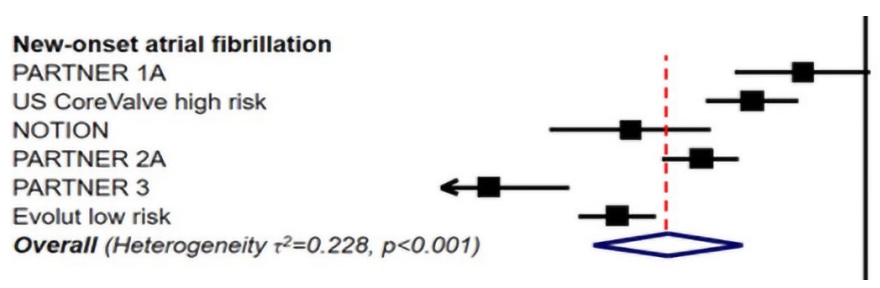

$0.71(0.49-1.02)$

$0.54(0.42-0.69)$

$0.28(0.18-0.43)$

$0.41(0.33-0.50)$

$0.13(0.09-0.20)$

$0.26(0.21-0.32)$

$0.34(0.23-0.51)$
$<0.001$

Figure 8 New-onset atrial fibrillation (24).

fewest number of particles (27). However, these differences have not translated into clinical events. Future studies will shed light to the definitive role of cerebral protection devices in TAVR.

\section{Permanent pacemaker and conduction abnormalities}

TAVR is known to cause clinically relevant conduction abnormalities due to the intimal anatomical relationship of the specialized electrical conduction system, particularly of the left bundle with the transcatheter valve landing zone and applied radial force (28).

In PARTNER 3, no statistical differences between TAVR and SAVR were observed regarding need for new permanent pacemaker insertion ( $6.5 \%$ vs. $4.0 \%)$, despite a $23.7 \%$ rate of new left bundle branch block in the TAVR arm compared to $8.0 \%$ in the SAVR arm. The Evolut low risk trial demonstrated a 30 -day requirement for permanent pacemaker of $17.4 \%$ in the TAVR group and $6.1 \%$ in the SAVR group. These findings are consistent with prior studies, which have noted an increase in permanent pacemaker insertion with self-expanding valves (29). In the SURTAVI study of intermediate-risk patients who were treated with a self-expanding CoreValve or Evolut $\mathrm{R}$ valve, the rate of pacemaker insertion was $25.9 \%$, compared to $6.6 \%$ in the SAVR group (95\% CI: 15.9-22.7) (12). In the CoreValve study of high-risk patients, the rate of pacemaker insertion at one year was $22.3 \%$ in the TAVR arm, compared to $11.3 \%$ in the SAVR $\operatorname{arm}(\mathrm{P}<0.001)(11)$.

The clinical consequences of pacemaker placement in TAVR patients are still controversial. It is known that increased right ventricular pacing burden leads to a higher risk of left ventricular dysfunction and worsening survival (29). Given the longer expected survival of patients enrolled in PARTNER 3 and Evolut low risk, the long-term implications and risk of pacemaker insertion will need to be closely monitored.

\section{Atrial fibrillation}

In both trials, the incidence of post-procedural new-onset atrial fibrillation (NOAF) in TAVR patients was significantly lower than in SAVR patients, from approximately 35-40\% to $5-8 \%$. In a recent meta-analysis of randomized clinical trials comparing TAVR and SAVR, all studies except for PARTNER 1A showed a significant reduction in NOAF with a hazards ratio (95\% CI) of $0.34(0.23-0.51), \mathrm{P}<0.001$ (Figure 8) (24). The lower NOAF risk may have an impact in subsequent mortality. Data from the STS/ACC TVT Registry found that the risk of death and stroke during hospital stay and at 1 year is higher in patients with NOAF, with the highest mortality in those discharged without anticoagulation (30). At present, there is no clear guideline recommendation for specific antithrombotic agents after TAVR. Upcoming trials, including patients with chronic atrial fibrillation, such as POPular-TAVI, AVANTAR and ATLANTIS will hopefully bring clarity to this field (31).

\section{Rehospitalization}

The PARTNER 3 trial reported lower overall rehospitalization rates with TAVR compared to SAVR at both 30 days $(3.4 \%$ vs. $6.5 \%)$ and 1 year $(7.3 \%$ vs. $11.0 \%)$. The Evolut Low Risk trial reported lower rehospitalization rates specifically for heart failure in the TAVR group at both time points (1.2\% vs. $2.5 \%$ and $3.2 \%$ vs. $6.5 \%)$ (Figure 9). These results not only indicate that TAVR in low-risk patients yields reduced rehospitalization rates compared to surgery, but also reflect decreased overall rehospitalizations compared to previous TAVR studies in higher-risk populations (Figure 10). In PARTNER 2, for example, 1-year rehospitalization rates after TAVR and SAVR were similar at $19.6 \%$ and $17.3 \%$, respectively.

\section{Quality of life}

Traditionally, TAVR has been associated with significant 

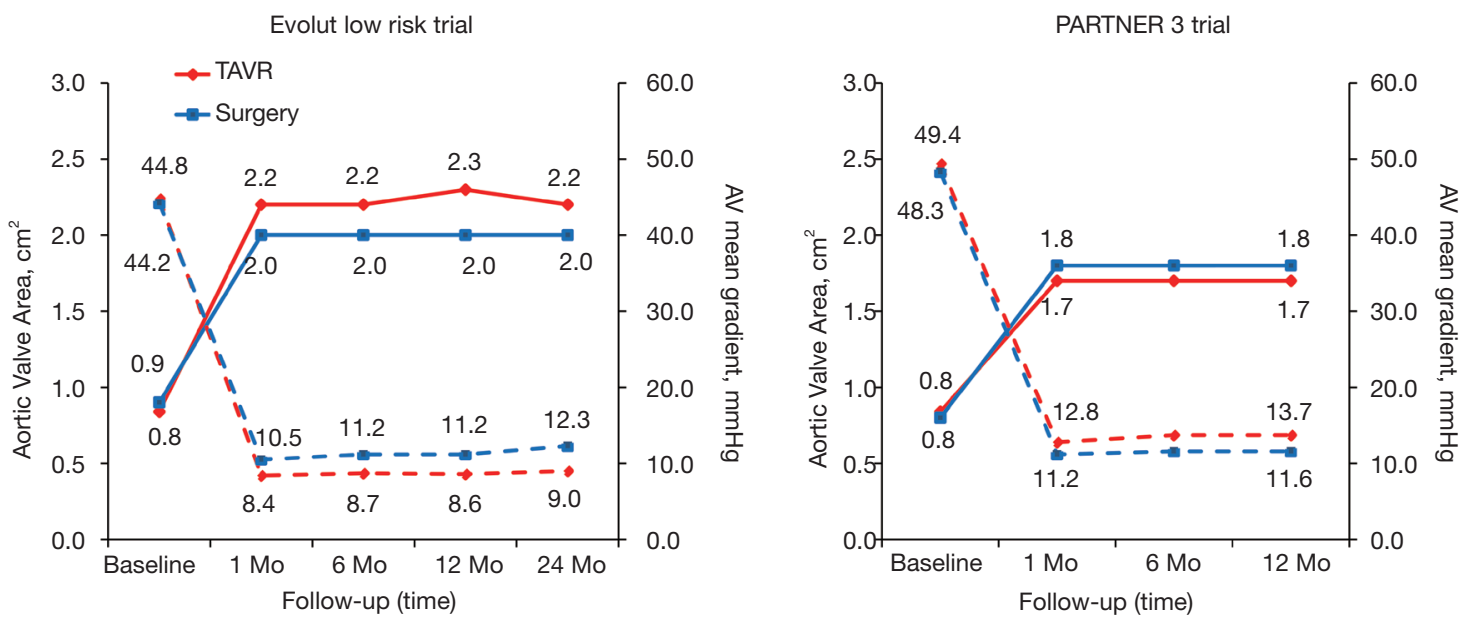

Figure 9 Valve hemodynamics in the PARTNER 3 and Evolut low risk trials.

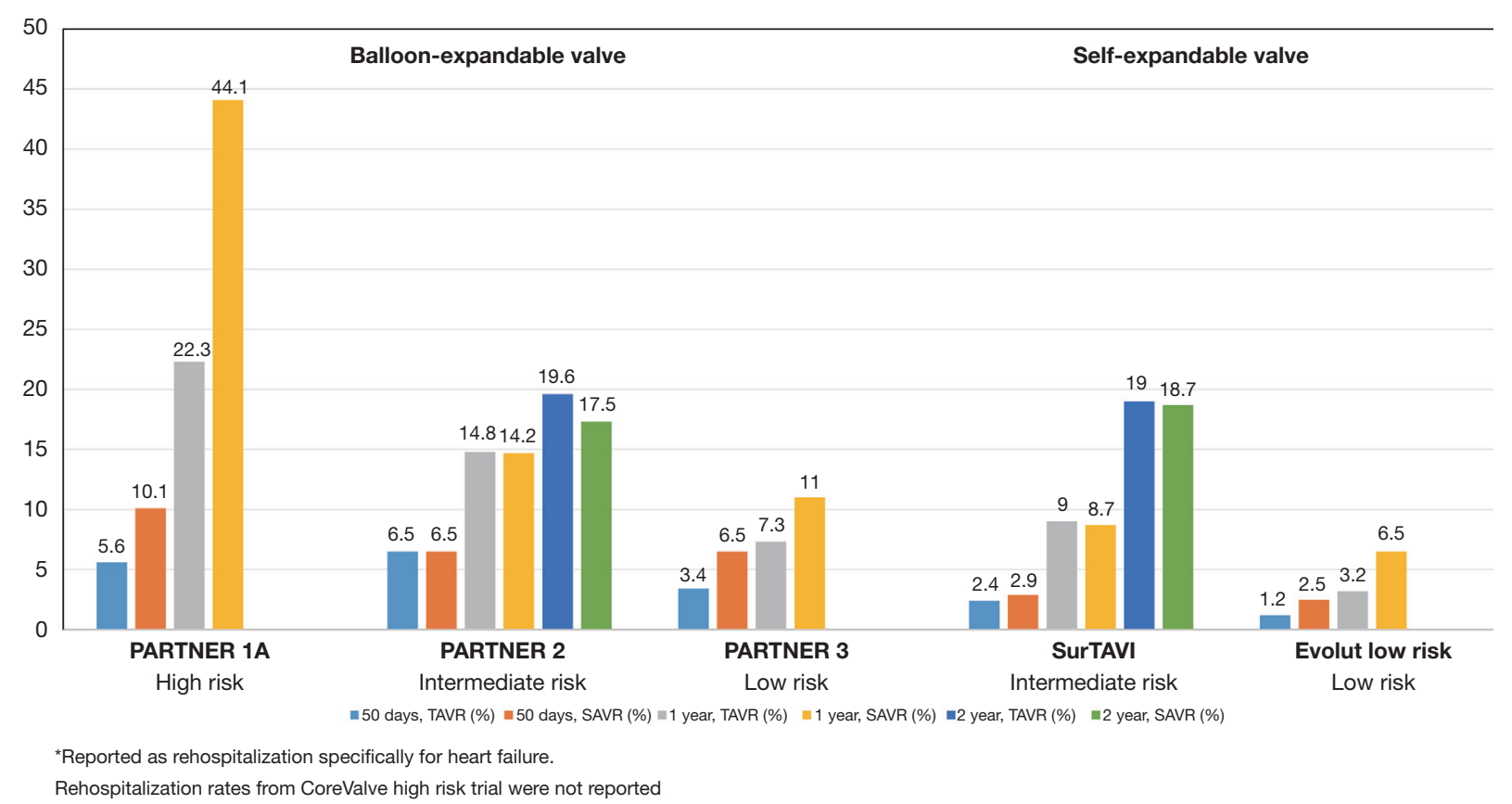

Figure 10 Rehospitalization.

improvement in quality of life scores, particularly in the short term (i.e., baseline to 30 days) (32). Because of the minimally invasive nature of TAVR and lack of thoracotomy/ sternotomy, post-TAVR patients typically experience a larger short-term improvement in health status as measured by the Kansas City Cardiomyopathy Questionnaire (KCCQ) at 30 days. However, these differences in KCCQ scores disappear after one year $(21,22)$.

In addition, both trials reported significant improvements in New York Heart Association (NYHA) class at 30 days and 1 year, regardless of treatment modality. In the Evolut low risk trial, the mean NYHA class improved by 0.9 after TAVR and 1.0 after SAVR from baseline to 12 months. In PARTNER 3, TAVR patients experienced greater improvement in NYHA class at 30 days, but the difference disappeared at 1 year. Only $17.7 \%$ in the TAVR group and $16.7 \%$ in the SAVR arm reported NYHA class II, III, and IV at 1 year. 


\section{Length of stay/cost}

As expected, TAVR resulted in a shorter median length of stay compared to SAVR (3.0 vs. 7.0 days for both trials) $(21,22)$. This finding is consistent with previous studies that have also suggested that TAVR patients are more likely to be discharged home rather than to a skilled nursing facility (33). Cost analyses have not been reported in the low-risk trials yet. However, it is expected that the shorter length of stay associated with TAVR will translate into significant cost savings during follow-up. A recent costeffectiveness analysis from the PARTNER 2 trial reported that although TAVR procedural costs were $\sim \$ 20,000$ higher than SAVR, total cost of index hospitalization was $\$ 4,155$ lower in patients receiving the SAPIEN S3 valve. This reduction was largely attributed to lower lengths of stays with TAVR (34). Beyond financial benefits, a decreased length of stay also reduces the potential for infections and other in-hospital complications. Longer hospital stays and delayed discharge are associated with greater risk of heart attack, stroke, and death after one year (35).

\section{Valve area and hemodynamics}

The PARTNER 3 and Evolut low risk trials enrolled patients with severe symptomatic aortic stenosis, defined by current guidelines as an aortic jet velocity of $\geq 4 \mathrm{~m} / \mathrm{s}$, or a mean aortic gradient of $\geq 40 \mathrm{mmHg}$. The presence of an aortic bicuspid valve was an exclusion criterion.

While the average left ventricular ejection fraction (LVEF) was $65.7 \% \pm 9.0 \%$ and $66.2 \% \pm 8.6 \%$ for the TAVR and SAVR groups respectively in PARTNER 3, asymptomatic patients with decreased LVEF ( $<50 \%)$ (AHA/ACC class C2) were also eligible for inclusion. In the Evolut low risk trial, the LVEF was somewhat lower at $61.7 \% \pm 7.9 \%$ for TAVR and $61.9 \% \pm 7.7 \%$ for SAVR.

The average baseline mean transvalvular aortic gradient was also very similar across the two different treatment modalities in both trials, although numerically higher in patients treated with transcatheter valves. Mean gradients in PARTNER 3 were $49.4 \pm 12.8$ and $48.3 \pm 11.8 \mathrm{mmHg}$ in the TAVR and SAVR groups, respectively. In Evolut low risk, mean gradients were $47.2 \pm 12.3$ and $46.7 \pm 12.2 \mathrm{mmHg}$ in the TAVR and SAVR groups, respectively. The average baseline aortic valve area of $0.8 \pm 0.2 \mathrm{~cm}^{2}$ amongst the surgical and transcatheter arms in both trials was the same.

After TAVR or SAVR, aortic valve hemodynamics was substantially improved from baseline in both groups
(Figure 9). Consistent with previous trials and possibly related to the nature of the supra-annular design of selfexpanding bioprostheses, lower transaortic mean gradients and larger aortic valve areas were observed after TAVR in the Evolut low risk trial $(11,12)$. At 24 months, the mean aortic gradient was $9.0 \mathrm{mmHg}$ in the TAVR group and $12.3 \mathrm{mmHg}$ in the SAVR group, while the mean aortic-valve area was 2.2 and $2.0 \mathrm{~cm}^{2}$, respectively. In the PARTNER 3 trial at 1 year, the mean aortic valve gradient was $12.8 \mathrm{mmHg}$ in the TAVR group and $11.2 \mathrm{mmHg}$ in the SAVR group, and the mean aortic-valve area was 1.7 and $1.8 \mathrm{~cm}^{2}$, respectively. It should be noted that in PARTNER 3 , the post-TAVR aortic valve area was slightly larger compared to PARTNER 1A (TAVR $1.6 \pm 0.5 \mathrm{~cm}^{2}$, SAVR $1.4 \pm 0.5 \mathrm{~cm}^{2}$ ) and PARTNER 2 (TAVR $1.6 \pm 0.4 \mathrm{~cm}^{2}$, SAVR $\left.1.4 \pm 0.4 \mathrm{~cm}^{2}\right)$.

At the same time, however, this is the first occurrence in the PARTNER series in which the aortic valve area is smaller and the mean aortic gradient is larger compared to post-SAVR patients. It is likely that the accumulation of knowledge and outcomes associated with prosthesispatient mismatch (PPM) led to a proactive effort to implant larger valves by highly experienced operators. Through the CoreValve, SURTAVI and Evolut trials, there has been a trend towards surgically implanting larger sized valves. The percentage of surgical patients receiving a valve smaller than $21 \mathrm{~mm}$ has decreased from $8.5 \%$, to $6.4 \%$, to $3.6 \%$, respectively, in the three trials. In PARTNER 3, only $2.9 \%$ of patients received a surgically implanted valve smaller than $21 \mathrm{~mm}$. While this data is not readily listed for PARTER 1 and 2, it is likely this same trend towards implanting larger sized valves surgically can be paralleled in this group of studies. This trend may account for the new hemodynamic changes seen in PARTNER 3 compared to prior studies.

\section{$P P M$}

PPM occurs when the effective orifice area (EOA) of the otherwise functional valve is too small in relation to a patient's body surface area. PPM is moderate when aortic valve area indexed (AVAi) to body surface area is less than $0.85 \mathrm{~cm}^{2} / \mathrm{m}^{2}$ and severe when less than $0.65 \mathrm{~cm}^{2} / \mathrm{m}^{2}$.

In the Evolut low risk trial, severe PPM occurred at 12 months in $1.8 \%$ of the patients in the TAVR group and in $8.2 \%$ in the SAVR group (22). In PARTNER 3, the rates of PPM at 30 days occurred in $8.3 \%$ in the TAVR group and $8.9 \%$ in the SAVR group (21).

PPM is a predictor of high transvalvular gradient, 


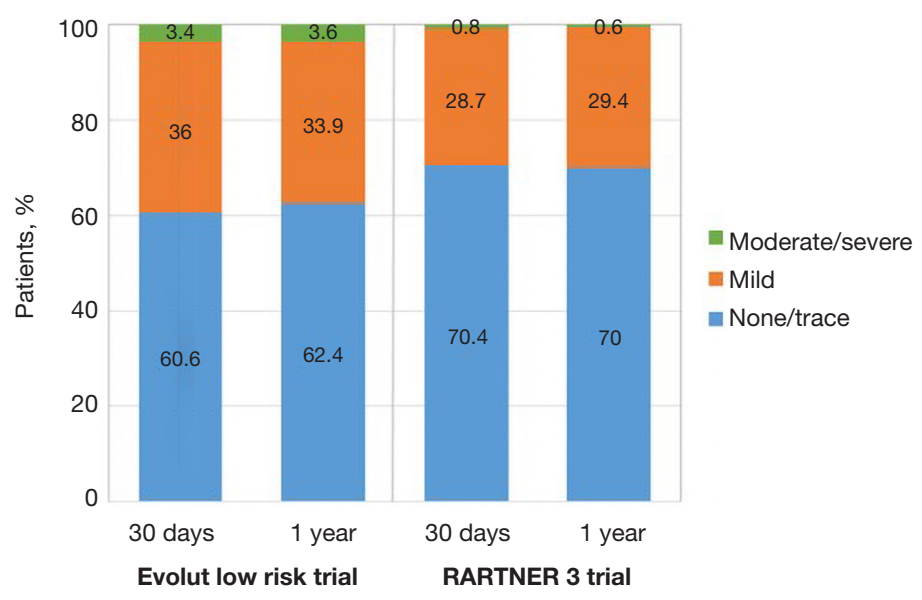

Figure 11 Paravalvular regurgitation over time in TAVR arms of the Evolut low risk and PARTNER 3 trial. TAVR, transcatheter aortic valve replacement.

structural valve degeneration, persistent LV hypertrophy, and carries an increased rate of cardiac events after aortic valve replacement. Especially in patients with reduced LVEF, PPM may decrease the likelihood of symptom improvement and LVEF recovery $(36,37)$. Surgical data indicates that patients who experienced PPM have limited functional class improvement, exercise tolerability (38), and a 1.5-2.0 fold increase in the risk of mortality and heart failure rehospitalization (39). In the STS/ACC TVT Registry, moderate and severe PPM occurred in $25 \%$ and $12 \%$ of cases, respectively. TAVR patients with severe PPM were noted to have higher mortality and rehospitalizations for heart failure at 1 year (40). Factors predisposing patients towards PPM include a smaller prosthesis $(\leq 23 \mathrm{~mm})$, valve in valve procedures, lower ejection fraction, atrial fibrillation, co-existing mitral/tricuspid regurgitation, as well as larger body surface area, younger age and female gender (40).

\section{$P V L$ and aortic regurgitation}

Moderate to severe PVL, defined as a $\geq 30 \%$ regurgitation, has been associated with increased mortality (41). In PARTNER 3, rates of moderate or severe PVL did not differ significantly between TAVR and SAVR $(0.8 \%$ and none, respectively, at 30 days; $0.6 \%$ and $0.5 \%$ at 1 year). In Evolut low risk, rates of moderate PVL were higher in the TAVR cohort $(3.4 \%$ vs. $0.6 \%$ at 1 year; $5.7 \%$ vs. $0 \%$ in 2 years). There were very few cases of severe PVL in the Evolut low risk trial (Figure 11).

Although the overall occurrence of moderate-to-severe
PVL has decreased over time with newer generation devices and more refined deployment technique, self-expanding valves are still more likely to have residual PVL compared with balloon-expandable valves. The most likely explanation for this finding is the polyethylene terephthalate outer skirt of the SAPIEN 3 valve which is designed to limit PVL. In contrast, the Evolut R transcatheter valve, used in three quarters of cases in the Low Risk trial, lacks a sealing skirt (22). New fully repositionable transcatheter valves with an adaptive seal design, such as the Lotus valve, can virtually eliminate residual PVL and may be an appealing choice for low-risk patients with longer life expectancy (42).

\section{TAVR for low risk patients and valve durability}

The durability of transcatheter heart valves is of utmost importance as the indication for TAVR has now been approved by the FDA for younger, low-risk patients with higher life expectancy. The biological valve tissue material of transcatheter and surgical valves is prone to degeneration. Therefore, long-term durability, along with factors which may influence long-term valve performance, such as valve hemodynamics, have become a major focus of investigation. The precise mechanisms of structural valve deterioration (SVD) have not been clearly identified but are likely to include tissue disruption or thickening due to a combination of mechanical stress, abnormal flow, collagen fiber deposition, tissue calcification, and shear stresses at the surface of valve leaflets $(43,44)$. There are limited data available on the durability and long-term hemodynamic performance of transcatheter prostheses. Predictors of 
hemodynamic deterioration include patient and procedural factors such as male gender, increased body mass index, small prosthesis size ( $\leq 23-\mathrm{mm}$ valve size), increased baseline aortic valve gradient, and severe PPM.

A recent single arm study in 200 low-risk patients undergoing TAVR showed that hypo-attenuated leaflet thickening (HALT) at 30 days occurs in 14\% of cases. Even though there is no definitive signal that leaflet thrombosis is associated with SVD, the presence of HALT is concerning because a numerically higher incidence of stroke was observed in patients with HALT (3.8\% vs. $1.9 \%$; $\mathrm{P}=0.53)$ (20).

To date, the incidence of clinically relevant SVD postTAVR has been very low (45) and similar to that reported for surgical bioprostheses (46). For example, the 5-year results of the PARTNER-1 trial showed no evidence of SVD, with mean transvalvular gradient of 10.7 and $10.6 \mathrm{mmHg}$ and aortic valve area of 1.6 and $1.5 \mathrm{~cm}^{2}$ in the TAVR and SAVR groups, respectively (6,7). Additionally, serial evaluations of the CoreValve system indicated no significant increase in transvalvular mean gradient over a 5 -year follow-up period (47). Lastly, 5-year results of the NOTION trial demonstrated that CoreValve was associated with larger prosthetic valve area $\left(1.7\right.$ vs. $\left.1.2 \mathrm{~cm}^{2}, \mathrm{P}<0.001\right)$ and lower mean gradient $(8.2$ vs. $13.7 \mathrm{mmHg}, \mathrm{P}<0.001)$ than SAVR in low-risk patients (48).

\section{Future directions}

The PARTNER 3 and Evolut low risk trials show that TAVR is associated with significantly lower incidence of disabling stroke, bleeding complications, acute kidney injury, and atrial fibrillation, but higher rates of aortic regurgitation and need for permanent pacemaker implantation, particularly with self-expanding valves, and similar mortality. Based largely on the results of these two trials, the FDA approved the use of TAVR in low-risk patients earlier in 2019, permitting use of the SAPIEN S3 and the CoreValve Evolut R and Evolut Pro valves.

Continued long-term clinical and echocardiographic follow-up will be crucial to definitively understand the durability of transcatheter heart valves and their different modes of failure. The results of imaging substudies will be very important to clarify the prognostic relevance of HALT.

It is worth mentioning that patients with bicuspid aortic valve disease, who comprise a significant proportion of younger low-risk patients with aortic stenosis, were excluded from the low-risk trials. These patients often have a non-circular annulus, predisposing to more PVL and risk of rupture (49). Additionally, these patients may have high stenotic burden, increasing the risk for periprocedural strokes.

In conclusion, in light of the results of the PARTNER 3 and Evolut low risk trials, the choice between transcatheter and surgical treatment for low-risk patients with aortic stenosis should be individualized, based on patient preference, anatomic factors, local surgical expertise and outcomes, underlying conduction abnormalities, and presence of comorbidities. It is clear that TAVR has a definite role in the management of these patients.

\section{Acknowledgments}

None.

\section{Footnote}

Conflicts of Interest: The authors have no conflicts of interest to declare.

Ethical Statement: The authors are accountable for all aspects of the work in ensuring that questions related to the accuracy or integrity of any part of the work are appropriately investigated and resolved.

\section{References}

1. Cribier A, Eltchaninoff H, Bash A, et al. Percutaneous Transcatheter Implantation of an Aortic Valve Prosthesis for Calcific Aortic Stenosis. Circulation 2002;106:3006-8.

2. Kundi H, Strom JB, Valsdottir LR, et al. Trends in Isolated Surgical Aortic Valve Replacement According to HospitalBased Transcatheter Aortic Valve Replacement Volumes. JACC Cardiovasc Interv 2018;11:2148-56.

3. Leon MB, Smith CR, Mack M, et al. Transcatheter aorticvalve implantation for aortic stenosis in patients who cannot undergo surgery. N Engl J Med 2010;363:1597-607.

4. Smith CR, Leon MB, Mack MJ, et al. Transcatheter versus surgical aortic-valve replacement in high-risk patients. $\mathrm{N}$ Engl J Med 2011;364:2187-98.

5. Leon MB, Smith CR, Mack MJ, et al. Transcatheter or Surgical Aortic-Valve Replacement in Intermediate-Risk Patients. N Engl J Med 2016;374:1609-20.

6. Kapadia SR, Leon MB, Makkar RR, et al. 5-year outcomes of transcatheter aortic valve replacement compared with standard treatment for patients with inoperable aortic stenosis (PARTNER 1): a randomised controlled trial. 
Lancet 2015;385:2485-91.

7. Mack MJ, Leon MB, Smith CR, et al. 5-year outcomes of transcatheter aortic valve replacement or surgical aortic valve replacement for high surgical risk patients with aortic stenosis (PARTNER 1): a randomised controlled trial. Lancet 2015;385:2477-84.

8. Webb JG, Doshi D, Mack MJ, et al. A Randomized Evaluation of the SAPIEN XT Transcatheter Heart Valve System in Patients With Aortic Stenosis Who Are Not Candidates for Surgery. JACC Cardiovasc Interv 2015;8:1797-806.

9. Thourani VH, Kodali S, Makkar RR, et al. Transcatheter aortic valve replacement versus surgical valve replacement in intermediate-risk patients: a propensity score analysis. Lancet 2016;387:2218-25.

10. Popma JJ, Adams DH, Reardon MJ, et al. Transcatheter aortic valve replacement using a self-expanding bioprosthesis in patients with severe aortic stenosis at extreme risk for surgery. J Am Coll Cardiol 2014;63:1972-81.

11. Adams DH, Popma JJ, Reardon MJ, et al. Transcatheter aortic-valve replacement with a self-expanding prosthesis. N Engl J Med 2014;370:1790-8.

12. Reardon MJ, Van Mieghem NM, Popma JJ, et al. Surgical or Transcatheter Aortic-Valve Replacement in Intermediate-Risk Patients. N Engl J Med 2017;376:1321-31.

13. Gleason TG, Reardon MJ, Popma JJ, et al. 5-Year Outcomes of Self-Expanding Transcatheter Versus Surgical Aortic Valve Replacement in High-Risk Patients. J Am Coll Cardiol 2018;72:2687-96.

14. Nishimura RA, Otto CM, Bonow RO, et al. 2017 AHA/ ACC Focused Update of the 2014 AHA/ACC Guideline for the Management of Patients With Valvular Heart Disease: A Report of the American College of Cardiology/ American Heart Association Task Force on Clinical Practice Guidelines. Circulation 2017;135:e1159-95.

15. Baumgartner H, Falk V, Bax JJ, et al. 2017 ESC/EACTS Guidelines for the management of valvular heart disease. Eur Heart J 2017;38:2739-91.

16. Thourani VH, Suri RM, Gunter RL, et al. Contemporary real-world outcomes of surgical aortic valve replacement in 141,905 low-risk, intermediate-risk, and high-risk patients. Ann Thorac Surg 2015;99:55-61.

17. D'Agostino RS, Jacobs JP, Badhwar V, et al. The Society of Thoracic Surgeons Adult Cardiac Surgery Database: 2018 Update on Outcomes and Quality. Ann Thorac Surg 2018;105:15-23.
18. Lange R, Bleiziffer S, Mazzitelli D, et al. Improvements in transcatheter aortic valve implantation outcomes in lower surgical risk patients: a glimpse into the future. J Am Coll Cardiol 2012;59:280-7.

19. Wenaweser P, Stortecky S, Schwander S, et al. Clinical outcomes of patients with estimated low or intermediate surgical risk undergoing transcatheter aortic valve implantation. Eur Heart J 2013;34:1894-905.

20. Waksman R, Rogers T, Torguson R, et al. Transcatheter Aortic Valve Replacement in Low-Risk Patients With Symptomatic Severe Aortic Stenosis. J Am Coll Cardiol 2018;72:2095-105.

21. Mack MJ, Leon MB, Thourani VH, et al. Transcatheter Aortic-Valve Replacement with a Balloon-Expandable Valve in Low-Risk Patients. N Engl J Med 2019;380:1695-705.

22. Popma JJ, Deeb GM, Yakubov SJ, et al. Transcatheter Aortic-Valve Replacement with a Self-Expanding Valve in Low-Risk Patients. N Engl J Med 2019;380:1706-15.

23. Pascual I, Carro A, Avanzas P, et al. Vascular approaches for transcatheter aortic valve implantation. J Thorac Dis 2017;9:S478-87.

24. Siontis GCM, Overtchouk P, Cahill TJ, et al. Transcatheter aortic valve implantation vs. surgical aortic valve replacement for treatment of symptomatic severe aortic stenosis: an updated meta-analysis. Eur Heart J 2019. [Epub ahead of print].

25. Kahlert P, Knipp SC, Schlamann M, et al. Silent and apparent cerebral ischemia after percutaneous transfemoral aortic valve implantation: a diffusion-weighted magnetic resonance imaging study. Circulation 2010;121:870-8.

26. Kapadia SR, Kodali S, Makkar R, et al. Protection Against Cerebral Embolism During Transcatheter Aortic Valve Replacement. J Am Coll Cardiol 2017;69:367-77.

27. Seeger J, Virmani R, Romero M, et al. Significant Differences in Debris Captured by the Sentinel DualFilter Cerebral Embolic Protection During Transcatheter Aortic Valve Replacement Among Different Valve Types. JACC Cardiovasc Interv 2018;11:1683-93.

28. Toggweiler S, Kobza R. Pacemaker implantation after transcatheter aortic valve: why is this still happening? J Thorac Dis 2018;10:S3614-9.

29. Tops LF, Schalij MJ, Bax JJ. The effects of right ventricular apical pacing on ventricular function and dyssynchrony implications for therapy. J Am Coll Cardiol 2009;54:764-76.

30. Vora AN, Dai D, Matsuoka R, et al. Incidence, Management, and Associated Clinical Outcomes of New- 
Onset Atrial Fibrillation Following Transcatheter Aortic Valve Replacement: An Analysis From the STS/ACC TVT Registry. JACC Cardiovasc Interv 2018;11:1746-56.

31. Sondergaard L, Sawaya FJ. Antithrombotic Management After Transcatheter Aortic Valve Replacement: More Questions Than Answers. JACC Cardiovasc Interv 2017;10:75-8.

32. Kim CA, Rasania SP, Afilalo J, et al. Functional status and quality of life after transcatheter aortic valve replacement: a systematic review. Ann Intern Med 2014;160:243-54.

33. Arora S, Strassle PD, Kolte D, et al. Length of Stay and Discharge Disposition After Transcatheter Versus Surgical Aortic Valve Replacement in the United States. Circ Cardiovasc Interv 2018;11:e006929.

34. Baron SJ, Wang K, House JA, et al. Cost-Effectiveness of Transcatheter Versus Surgical Aortic Valve Replacement in Patients With Severe Aortic Stenosis at Intermediate Risk. Circulation 2019;139:877-88.

35. Wayangankar SA, Elgendy IY, Xiang Q, et al. Length of Stay After Transfemoral Transcatheter Aortic Valve Replacement: An Analysis of the Society of Thoracic Surgeons/American College of Cardiology Transcatheter Valve Therapy Registry. JACC Cardiovasc Interv 2019;12:422-30.

36. Flameng $W$, Herregods MC, Vercalsteren $M$, et al. Prosthesis-patient mismatch predicts structural valve degeneration in bioprosthetic heart valves. Circulation 2010;121:2123-9.

37. Poulin F, Yingchoncharoen T, Wilson WM, et al. Impact of Prosthesis-Patient Mismatch on Left Ventricular Myocardial Mechanics After Transcatheter Aortic Valve Replacement. J Am Heart Assoc 2016. doi: 10.1161/ JAHA.115.002866.

38. Pibarot P, Dumesnil JG. Prosthesis-patient mismatch: definition, clinical impact, and prevention. Heart 2006;92:1022-9.

39. Pibarot P, Magne J, Leipsic J, et al. Imaging for Predicting and Assessing Prosthesis-Patient Mismatch After Aortic Valve Replacement. JACC Cardiovasc Imaging 2019;12:149-62.

40. Herrmann HC, Daneshvar SA, Fonarow GC, et al.

Cite this article as: Braghiroli J, Kapoor K, Thielhelm TP, Ferreira T, Cohen MG. Transcatheter aortic valve replacement in low risk patients: a review of PARTNER 3 and Evolut low risk trials. Cardiovasc Diagn Ther 2020;10(1):59-71. doi: 10.21037/cdt.2019.09.12
Prosthesis-Patient Mismatch in Patients Undergoing Transcatheter Aortic Valve Replacement: From the STS/ ACC TVT Registry. J Am Coll Cardiol 2018;72:2701-11.

41. Sponga S, Perron J, Dagenais F, et al. Impact of residual regurgitation after aortic valve replacement. Eur J Cardiothorac Surg 2012;42:486-92.

42. Feldman TE, Reardon MJ, Rajagopal V, et al. Effect of Mechanically Expanded vs. Self-Expanding Transcatheter Aortic Valve Replacement on Mortality and Major Adverse Clinical Events in High-Risk Patients With Aortic Stenosis: The REPRISE III Randomized Clinical Trial. JAMA 2018;319:27-37.

43. Cote N, Pibarot P, Clavel MA. Incidence, risk factors, clinical impact, and management of bioprosthesis structural valve degeneration. Curr Opin Cardiol 2017;32:123-9.

44. Dvir D, Bourguignon T, Otto CM, et al. Standardized Definition of Structural Valve Degeneration for Surgical and Transcatheter Bioprosthetic Aortic Valves. Circulation 2018;137:388-99.

45. Blackman DJ, Meredith IT, Dumonteil N, et al. Predictors of Paravalvular Regurgitation After Implantation of the Fully Repositionable and Retrievable Lotus Transcatheter Aortic Valve (from the REPRISE II Trial Extended Cohort). Am J Cardiol 2017;120:292-9.

46. Rodriguez-Gabella T, Voisine P, Puri R, et al. Aortic Bioprosthetic Valve Durability: Incidence, Mechanisms, Predictors, and Management of Surgical and Transcatheter Valve Degeneration. J Am Coll Cardiol 2017;70:1013-28.

47. Barbanti M, Petronio AS, Ettori F, et al. 5-Year Outcomes After Transcatheter Aortic Valve Implantation With CoreValve Prosthesis. JACC Cardiovasc Interv 2015;8:1084-91.

48. Thyregod HGH, Ihlemann N, Jorgensen TH, et al. FiveYear Clinical and Echocardiographic Outcomes from the Nordic Aortic Valve Intervention (NOTION) Randomized Clinical Trial in Lower Surgical Risk Patients. Circulation 2019. [Epub ahead of print]

49. Eagle KA. TAVR Safe in Patients with Bicuspid Aortic Valve: NCDR Late-Breaking Study. American College of Cardiology 2019. 